\title{
Comparison of dosimetric data of bone marrow between standard IMRT and bone marrow sparing IMRT in carcinoma cervix
}

\author{
Lucy Pattanayak, Samir Mohanta, Sasmita Panda, Manoranjan Mohapatra \\ Acharya Harihar Post Graduate Institute of Cancer, Manglabag, Cuttack, Odisha, India
}

\begin{abstract}
Background: The aim of the study was to assess the dosimetric comparison of bone marrow between standard IMRT(SDIMRT) and bone marrow sparing IMRT (BMS-IMRT) among carcinoma cervix patients who underwent radical or adjuvant chemoradiation in a tertiary cancer center.

Materials and methods: Forty eligible patients of histo-pathologically proven carcinoma cervix were enrolled in the study that was randomized on a 1:1 basis between SD-IMRT and BMS-IMRT from July 2018 to October 2019. The whole pelvis, bilateral femoral heads, and upper $1 / 3^{\text {rd }}$ femur were contoured using the whole bone technique as a surrogate marker for the bone marrow. In both arms, V10, V20, and V40, bone marrow was noted along with mean, maximum, minimum dose, and total volume. DVH for the bone marrow in both arms was compared using the unpaired student t-test.

Results: We found no significant difference in the mean of various parameters in SD-IMRT arm vs. BMS IMRT arm - for the bone marrow: V10 ( $89 \pm 4.3 \%$ vs. $86.7 \pm 3.7 \%)$, V20 (73.2 $\pm 5.3 \%$ vs. $73.1 \pm 4.5 \%)$, V40 ( $23.9 \pm 5.4 \%$ vs. $26.6 \pm 7.4 \%)$ and, similarly, for mean dose $(28.1 \pm 3.5 \%$ vs. $28.1 \pm 1.8 \%)$, maximum dose $(53.4 \pm 0.58 \%$ vs. $53.2 \pm 0.58 \%)$, minimum dose $(0.33 \pm 0.18 \%$ vs. $0.38 \pm 0.38 \%)$, total volume ( $961 \pm 110$ cc vs. $901 \pm 152$ cc).

Conclusion: This study shows no statistically significant difference in dosimetry between the two groups, which suggests that SD-IMRT spares the bone marrow adequately. Therefore, the need for BMS-IMRT using the present contouring technique does not give any added advantage over SD-IMRT. However, large sample size, other novel contouring technique, and multivariate analysis are needed to reach a definite conclusion.
\end{abstract}

Key words: bone marrow sparing IMRT; pelvic radiation; carcinoma cervix

Rep Pract Oncol Radiother 2021;26(6):976-983

\section{Introduction}

Cervical cancer remains the most common gynecological malignancy worldwide and the fourth most common malignancy in women [1]. About 96,922 new cases are diagnosed annually in India (estimated in 2018). Cervical cancer is ranked as the fourth leading cause of cancer in women with an average age of 53 years at diagnosis [2]. Concurrent chemoradiation is the treatment of choice for stage IB2 and stage IIB-IVA. Radiotherapy is delivered through an external beam to the pelvis, which takes care of the regional nodal basin and the central disease (cervix, vagina, and medial parametria).

In contrast, brachytherapy is used for the treatment of central disease only. More than $50 \%$ of the

Address for correspondence: Dr. Lucy Pattanayak, Associate Professor, Dept. of Radiation Oncology, Acharya Harihar Post Graduate Institute of Cancer, Manglabag, Cuttack-753007, Odisha, India. Phone: +91- 9937028362; e-mail: lucypattanayak2007@gmail.com 
body bone marrow (BM) is located in the pelvic bone, proximal femora, and lower lumbar spine $[3,4]$. In conventional pelvic RT, these areas are included in the treatment volume that leads to hematological toxicities [5], and bone marrow regeneration varies with radiation doses [6-9]. Besides, concomitant chemotherapy further augments bone marrow toxicity. Thus, reducing the BM dose may decrease radiation toxicities, thereby enabling improved chemotherapy delivery and, consequently, treatment efficacy.

Since most carcinoma cervix patients in India suffer from Iron deficiency anemia, BM toxicity due to irradiation further deteriorates the hematological profile during treatment [10]. Higher hemoglobin level Cervical cancer remains the most common gynecological malignancy worldwide and the fourth most common malignancy in women [1].

Higher hemoglobin level generally has a favorable effect on the patient's well-being, energy level, and impacts tumor radiosensitivity. Hypoxic tumors are more likely to recur locoregionally than well-oxygenated tumors regardless of whether surgery or radiotherapy is the primary local treatment.

Radiotherapy techniques and equipment types have evolved in terms of conformity, dose-escalation, and normal tissue sparing for the past ten decades. Intensity-modulated radiation therapy (IMRT) can achieve greater conformity by optimally modulating the intensity of individual beams with sharper fall-off dose at the target boundaries, thereby reducing the toxicity of normal tissue. This dose conformity effect of IMRT can significantly reduce the $\mathrm{BM}$ dose as compared to $3 \mathrm{D}$ conformal radiation (3DCRT) therapy more significantly sparing nearby critical structures [11]. So, even without intentional BM sparing, a standard IMRT (SD-IMRT) is associated with a substantial reduction in BM irradiation dose and results in reduced hematologic toxicity (HT) compared with conventional whole pelvic RT. A bone marrow sparing IMRT (BMS-IMRT) plans are optimized to limit BM irradiation compared to SD-IMRT [12]. With the evolution of varying contouring techniques, like whole bone IMRT(WB-IMRT) and free hand IMRT(FH-IMRT), studies have shown a further reduction in dose to the bone marrow in the pelvis [13]. The present study's objective was to evaluate the advantage of BMS-IMRT over SD-IMRT in the treatment of carcinoma cervix undergoing chemo- radiation. In this study, the whole bone (WB) contouring technique was used as a surrogate marker for BM.

\section{Materials and methods}

This study was conducted in our post-graduate department of radiation oncology at Acharya Harihar Regional Cancer Centre (AHRCC), Cuttack, Odisha, India, from July 2018 to October 2019.

\section{Study design}

It was a hospital-based prospective, double-blinded, randomized study where 40 pathologically confirmed carcinoma cervix patients (FIGO stage IIB-IVA) with Karnofsky Performance Score (KPS) more than 70 with radical concurrent chemoradiation were included. All the included participants had histopathologically confirmed squamous cell carcinoma, were aged above 18 years, and had no prior chemoradiation history. Out of 40 patients, 20 were treated with SD-IMRT, and the remaining patients were treated with BMS-IMRT.

Patients with prior pelvic irradiation, KPS $\leq 70$, having evidence of distant metastasis, patients with synchronous tumors, and having any other malignancies in the past were excluded from the study. Besides, we had excluded pregnant patients, patients requiring paraaortic field irradiation, collagen vascular disease patients, renal and hepatic impaired patients, and the patients who underwent a hysterectomy from this investigation.

\section{Methodology}

After receiving an institutional ethics committee approval (93-IEC-AHRCC), we initiated this study at the department of radiation oncology, Acharya Harihar Regional Cancer Centre (AHRCC), Cuttack, Odisha, India. Detailed clinical history and baseline characteristics like age, performance status, biopsy, and routine hematological examination reports were evaluated. After a thorough systemic, pelvic examination, metastatic workup, and imaging, final FIGO staging was done. All the patients recruited in the study were randomized into two groups: SD-IMRT vs. BMS-IMRT (Fig. 1), on an odd-even numbering basis. Informed written consent was taken from all the participants and who were then taken for the treatment procedure. Both arms received concurrent chemotherapy cisplatin 


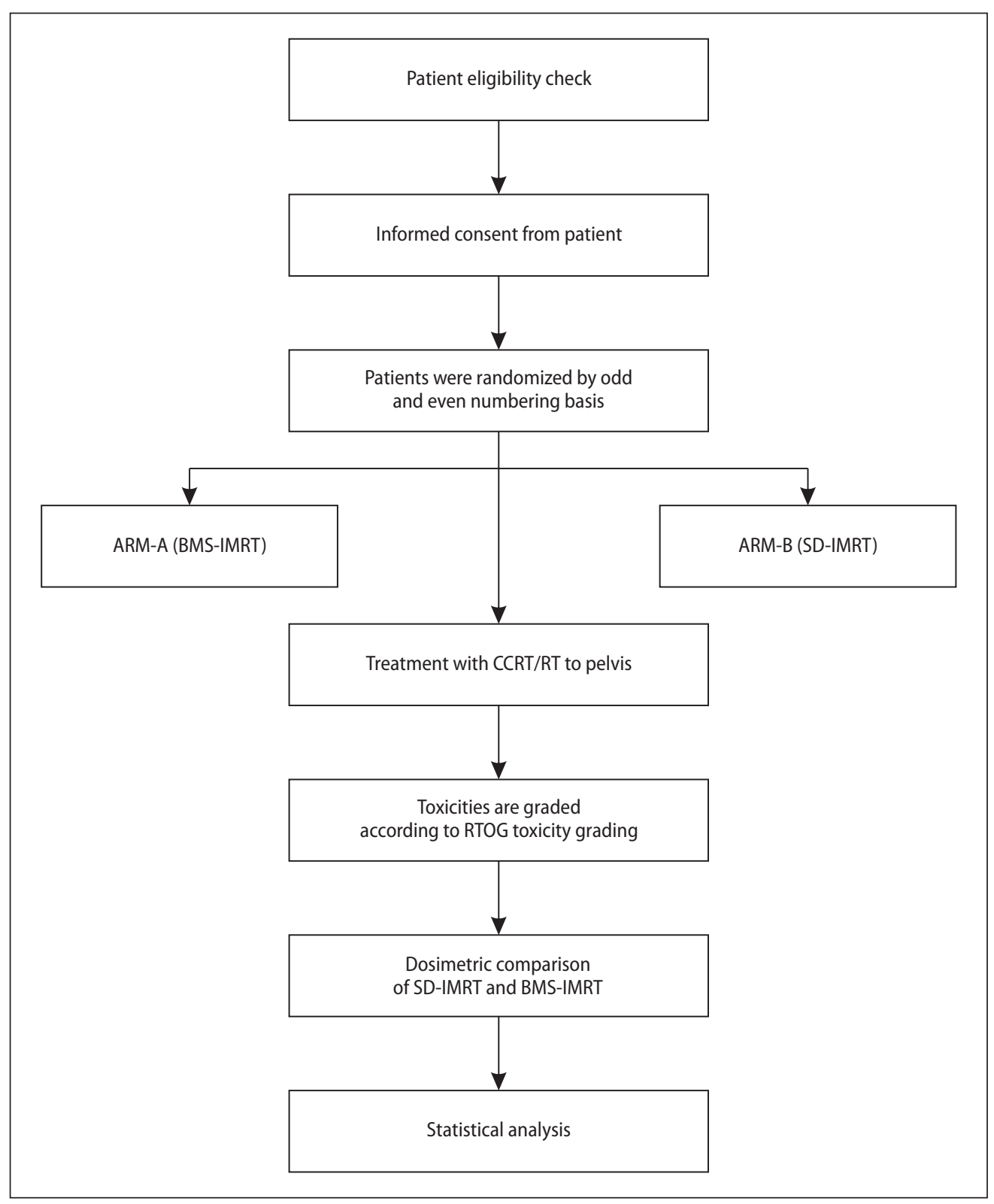

Figure 1. Study design flow diagram

$40 \mathrm{mg}$ per $\mathrm{m}^{2}$, a maximum of $60 \mathrm{mg}$ as a weekly regimen. A dose of $50 \mathrm{~Gy}$ in 25 fractions external beam RT was given to all patients, followed by HDR intracavitary brachytherapy. The entire treatment procedure was completed within eight weeks. The baseline hemogram $(\mathrm{Hb})$, total leukocyte counts (TLC), and total platelet count were recorded for all patients for future reference and were reviewed every week.

We followed a strict and uniform bowel bladder protocol for all patients before computed tomography (CT) simulation. Bowel preparation was done with an enema one day before CT simulation.
The patients were instructed to drink $500 \mathrm{ml}$ of water 30 minutes before simulation, were positioned supine with the arm abducted overhead, and immobilized with a rigid thermoplastic mask. One radio-opaque marker was placed at the introitus to mark the lower limit, and three radio-opaque markers were placed over the thermoplastic mask to delineate the CT isocenter. Intravenous (IV) contrast Iohexol was administered using proper IV cannula (20 Fr Gauze) with a dose of $1 \mathrm{~mL} / \mathrm{m}^{2}$ just before simulation for better delineation of the vessels and contrast enhancement of the disease. The CT scan was then acquired with slice thickness of 5 
$\mathrm{mm}$ from $\mathrm{D} 10-11$ of the vertebra to mid-thigh. The same bowel and bladder protocol was repeated each time before treatment.

\section{Target volume delineation}

According to PGI (Post Graduate Institute, Chandigarh) guideline ${ }^{(14)}$, we defined the target volume. For consistency, all contours were reviewed and edited by the unit head. A $5 \mathrm{~mm}$ margin was generated for setup error over clinical target volume (CTV) to get the planning target volume (PTV).

\section{Normal tissue definition}

Normal tissue included the small bowel, bladder, rectum, femoral head of both sides contoured using RTOG guidelines for OAR [15]

\section{BM delineation}

The external contour of all bones within the pelvis was delineated on planning CT scan as a surrogate marker for $\mathrm{BM}$, also called the whole bone (WB) technique. This technique was chosen over the free hand $(\mathrm{FH})$ technique because it was easy to perform, no other modalities were needed, and inter-observer variation was minimal. The pelvic bone, which was contoured, included the bilateral ilium, ischium, acetabulum, femoral heads up to the ischial tuberosity, and also the entire sacrum, vertebral body contained in the planning treatment volume (usually L5). In SD-IMRT, bone marrow constraints were not given, whereas, in BMS-IMRT, constraints to the $\mathrm{BM}$ were given along with all the OARs.

\section{Constraints given for organs at risk (OARs)} Constraints given for OARs included:

- bowel bag V45 < 195cc (V45: volume of bowel receiving $45 \mathrm{~Gy}$ )

- rectum V50 $<50 \%$ of total volume (V50: volume of rectum receiving $50 \mathrm{~Gy}$ )

- bladder V50 < 50\% of total volume (V50: volume of bladder receiving $50 \mathrm{~Gy}$ )

- bone marrow: V10 < 95\% of BM volume, $\mathrm{V} 20<90 \%$ of BM volume, $\mathrm{V} 40<45 \%$ of BM volume (V10, V20, V40 - volume of bone marrow receiving 10, 20 and 40 Gy respectively) [19-21].

\section{Treatment planning}

We used the "MONACO" planning system to plan the treatment. The inverse plans were gen- erated with the placement of multiple coplanar beams and the $6 \mathrm{MV}$ photon beam. The best plan was accepted from among the generated plans. All plans were normalized to cover $95 \%$ of the PTV with at least $95 \%$ of the prescription dose. Then, the dose-volume histogram (DVH) was analyzed, and various parameters like V10, V20, V40, Mean, Maximum, Minimum dose of BM, and the total volume of BM were noted.

\section{Dose prescription}

External beam radiotherapy dose to the PTV was $50 \mathrm{~Gy} / 25$ fractions, $2 \mathrm{~Gy} /$ fraction, five fractions/week over five weeks in both treatment arms were given.

\section{Dose delivery}

Patients underwent the same bowel and bladder preparation before every fraction, similar to CT simulation, which included bowel preparation with enema and bladder preparation with $500 \mathrm{ml}$ of water 30 minutes before treatment. Cone-beam CT (CBCT) was performed every week to check positional errors.

\section{Statistical analysis}

In both arms, the dose received by the bone marrow was analyzed using the parameters like V10, V20, V40, mean dose, maximum dose, minimum dose, and the total volume of bone as a surrogate marker for the bone marrow. The data were entered into a Microsoft Excel spreadsheet and analyzed using IBM SPSS Statistics for Windows, version 24 (IBM Corp., Armonk, NY, USA). A student's

Table 1. Age and stage-wise distribution of patients in each treatment arm

\begin{tabular}{|c|c|c|c|}
\hline Age group [yrs] & SD-IMRT & BMS-IMRT & Total \\
\hline$<40$ & 5 & 0 & 5 \\
\hline $40-49$ & 6 & 9 & 15 \\
\hline $50-59$ & 6 & 10 & 16 \\
\hline $60-69$ & 3 & 1 & 4 \\
\hline$\geq 70$ & 0 & 0 & 0 \\
\hline \multicolumn{4}{|l|}{ Stage } \\
\hline IIB & $6(30 \%)$ & $8(40 \%)$ & $14(35 \%)$ \\
\hline IIIB & $14(70 \%)$ & $12(60 \%)$ & $26(65 \%)$ \\
\hline Total & 20 & 20 & 40 \\
\hline
\end{tabular}

SD-IMRT - standard intensity modulated radiation therapy BMS-IMRT - bone marrow sparing intensity modulated radiation therapy 
unpaired t-test was performed to interpret the results, and a p-value of less than 0.05 was considered statistically significant.

\section{Results}

The median age of patients at presentation was 47.5 years and 50 years in SD-IMRT and BMS-IMRT treatment groups. The groups in comparison were identical (stage-wise distribution for both the arms) (Tab. 2).

In both arms, the dose received by OARs like the rectum, bladder, head of the femur, bowel bag were achieved as per dose constraints provided during inverse planning based on RTOG toxicity guidelines. The average volume of PTV receiving $95 \%$ of the prescribed dose was $96.4 \%$ in SD-IMRT and $96.5 \%$ in the BMS-IMRT arm. Figure 2 shows that the blue color represents PTV, and the pink color represents the whole pelvic bone as the surrogate marker for the bone marrow. Similarly, Figure 3 shows the dose distribution of SD-IMRT and BMS-IMRT. Visually, we find a similar dose distribution pattern in both SD-IMRT and BMS-IMRT.

We found no significant difference in all the analyzed parameters between both groups (Tab. 2). This means that the dose received by BM in the arm where we intended to limit the dose to BM by giving constraints through inverse planning was

Table 2. Comparison of bone marrow dose in detail between two arms

\begin{tabular}{|l|c|c|c|}
\hline \multirow{2}{*}{ V10 } & \multicolumn{2}{|c}{ Technique } & \multicolumn{2}{c}{ Mean \pm SD } & p-value \\
\hline \multirow{2}{*}{ V20 } & SD-IMRT & $89.04 \pm 4.33$ & \multirow{2}{*}{0.8} \\
\cline { 2 - 3 } & BMS-IMRT & $86.77 \pm 3.73$ & \\
\hline \multirow{2}{*}{ V40 } & SD-IMRT & $73.2 \pm 5.3$ & \multirow{2}{*}{0.74} \\
\cline { 2 - 3 } & BMS-IMRT & $73.13 \pm 4.5$ & \\
\hline \multirow{2}{*}{ Mean dose } & SD-IMRT & $23.97 \pm 5.4$ & \multirow{2}{*}{0.14} \\
\cline { 2 - 3 } & BMS-IMRT & $26.62 \pm 7.43$ & \\
\hline \multirow{2}{*}{ Maximum dose } & SD-IMRT & $28.16 \pm 3.49$ & \multirow{2}{*}{0.96} \\
\cline { 2 - 3 } & BMS-IMRT & $28.12 \pm 1.86$ & \\
\hline \multirow{2}{*}{ Minimum dose } & SD-IMRT & $53.41 \pm 0.58$ & \multirow{2}{*}{0.37} \\
\cline { 2 - 3 } & BMS-IMRT & $53.24 \pm 0.58$ & \\
\cline { 2 - 3 } Bone volume [cC] & SD-IMRT & $0.33 \pm 0.18$ & \multirow{2}{*}{0.61} \\
\cline { 2 - 3 } & BMS-IMRT & $0.38 \pm 0.38$ & \\
\cline { 2 - 3 } & SD-IMRT & $961.1 \pm 110.5$ & \multirow{2}{*}{0.16} \\
\hline
\end{tabular}

SD-IMRT — standard intensity modulated radiation therapy; BMS-IMRT — bone marrow sparing intensity modulated radiation therapy

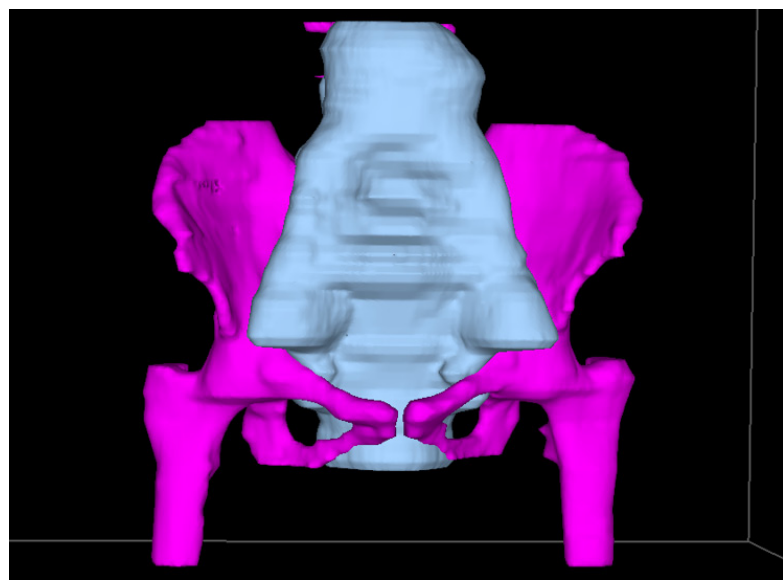

Figure 2. Blue color depicts planning target volume (PTV), and pink color represents the whole pelvic bone as a surrogate marker for bone marrow (BM)
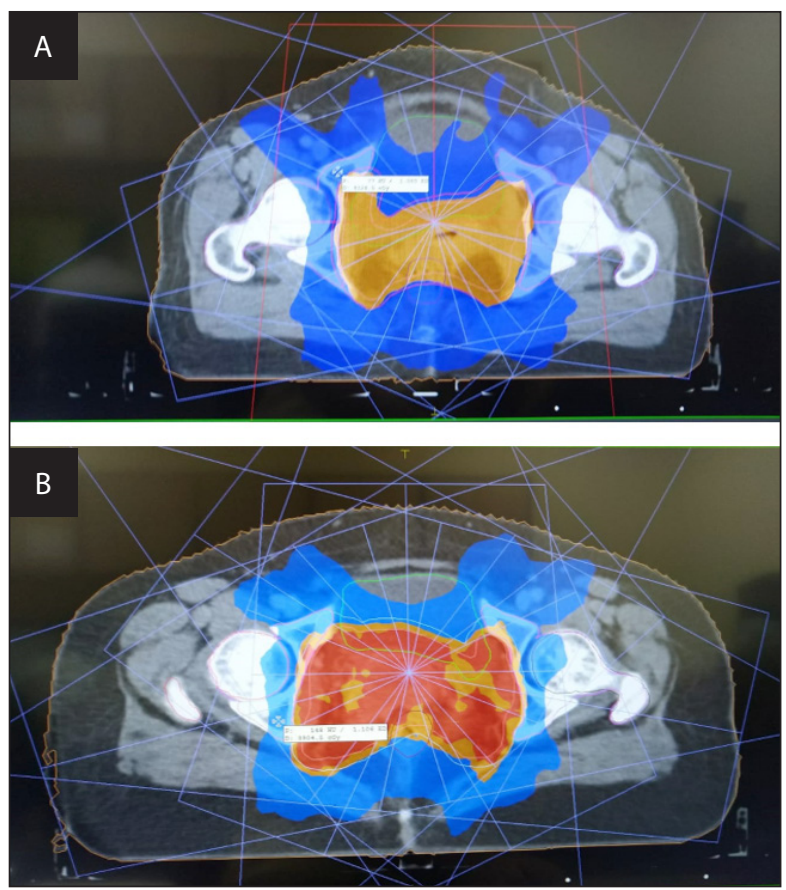

Figure 3. Dose distribution in standard intensity modulated radiation therapy (SD-IMRT) (A) and bone marrow sparing IMRT (BMS-IMRT) (B)

similar to the arm where no constraints were given to the bone marrow during planning. Hence, it indicates that the standard IMRT, as such, limits the dose to the bone marrow similarly without hampering the dose to the target volume. Besides, Figure 4 represents the dose-volume histogram of bone marrow sparing IMRT and standard IMRT of one patient from each arm. The two graphs show a similar pattern of percentage volume receiving 


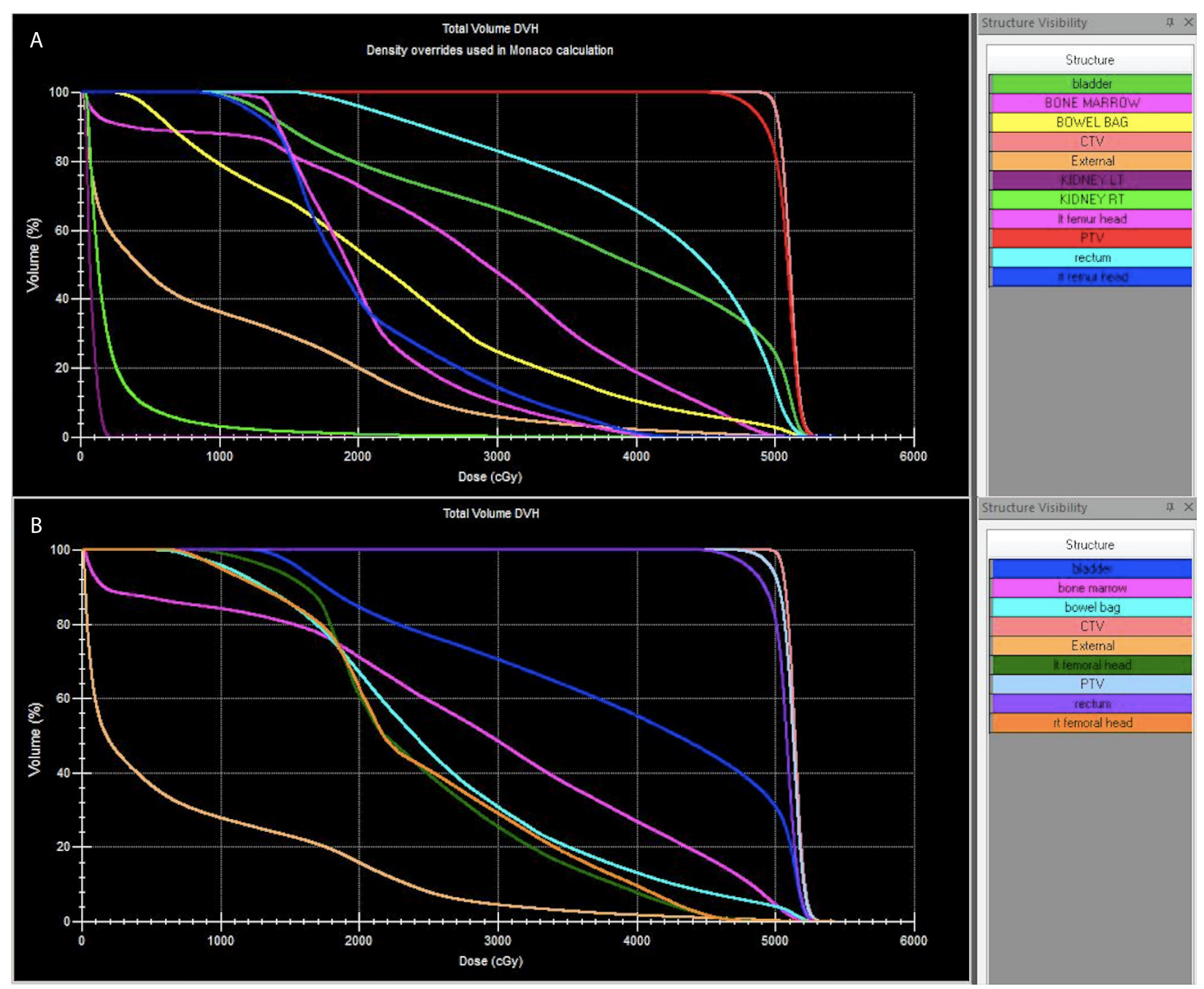

Figure 4. Dose volume histogram (DVH) of both arms, one from each arm. A. DVH of a patient with bone marrow sparing IMRT (BM-IMRT). B. DVH of a patient with standard IMRT (SD-IMRT)

$10 \mathrm{~Gy}, 20 \mathrm{~Gy}$, and $40 \mathrm{~Gy}$ of the bone marrow represented as a pink line.

\section{Discussion}

This study's objective was to compare the dosimetry between two subsets of patients using different techniques of contouring, i.e., BMS-IMRT vs. SD-IMRT, among those undergoing concurrent chemoradiation as a treatment in locally advanced carcinoma cervix. Mell et al. reported that pelvic $\mathrm{BM}$ volume receiving low-dose radiation (V10, V20) is less likely to suffer from acute HT in patients undergoing concurrent chemiradiation 16. They took the whole bone contouring tool for contouring bone marrow to eliminate inter-observer variation. Bone marrow is extremely radiosensitive, with histopathologic changes evident in doses as low as 4 Gy. However, starting at $10 \mathrm{~Gy}$, progressively, more significant changes are seen, including dilated sinusoids, acute hemorrhage, and the reduction of precursor cells. With doses of more than $50 \mathrm{~Gy}$, complete hypoplasia can occur without regeneration [16]. So, V10 is the most important predicting factor for bone marrow toxicity. Thus, reducing the V10 dose may decrease in hematological toxicities. Further, there is also a correlation between Haematological toxicity in V40 of BM. When $\mathrm{BM} \mathrm{V} 40>40 \%$, there is a higher hematological toxicity profile $[16,17]$. In our study, DVH parameters for V10, V20, V40 were $89.04 \pm 4.3,73.2 \pm 5.3$, and $23.97 \pm 5.4$ for the SD-IMRT arm, respectively. Similarly, for the BMS-IMRT group, the values were $86.77 \pm 3.7,73.1 \pm 4.5$, and $26.2 \pm 7.4$, respectively.

The whole bone comprises bone and the cavity which has the active and inactive yellow marrow. 
This cavity cannot be further differentiated on CT alone images; therefore, the whole bone was taken as a surrogate marker for the bone marrow. In this study, it was clear that there is no significant difference in DVH parameters in the two arms. The V10 in both arms is below 90\%. Similarly, V40 was also less than $30 \%$ in both arms. In this study, we found no additional advantage of constraints to the bone marrow (i.e., V10 < 95\% and V40<40\%) in BMS-IMRT compared to SD-IMRT where no bone marrow constraint was given.

In developing countries, most patients presenting with locally advanced stages require intensive treatments with a more prevalent problem of malnutrition, anemia, and low body mass index [18]. Hence, it would make HT more common and challenging to deal with, especially with intensified treatment schedules.

Our priority was to achieve the desired target coverage. $95 \%$ of the prescribed volume covered 96.5\% (mean) in the BMS-IMRT arm and 96.4\% (mean) SD-IMRT arm. Other OARs like the rectum, bladder, bilateral femoral head, and small bowel dose was within the prescribed limit.

McGuire et al. [22] took FLT-PET to delineate the active bone marrow volume. However, in this study, we took the whole pelvic bone as a surrogate marker for the bone marrow. The active bone marrow volume is less than the whole bone marrow volume. So low dose volume like V5 is more important when OAR is only the active bone marrow. However, in our study, as we took the whole pelvic bone plus the upper third of the femur as a surrogate marker for the bone marrow, we took V10 as the constraints parameter.

In our institution, around 80 patients are treated with radiation on a $6 \mathrm{MV}$ linac (mostly 3DCRT) and around 70 patients are treated with radiation on a high energy linac (IMRT and VMAT) per day. People have to wait for a period of 1 month in queue to get the start dose of radiation. In such a busy setup it is very difficult to opt bone marrow contouring for all patients receiving pelvic IMRT. WHO recommendation is 1 teletherapy machine per 1 million population. Hence, we need around 1250 teletherapy units in India, whereas we have only 545 out of which there are only 365 Linac machines[23]. We have a shortfall of nearly 700 teletherapy units. Thus, it is evident that most of the centers in India have a similar scenario in the patient flow. Hence, adapting BMS-IMRT which is a time consuming technique and has no added advantage over SD-IMRT is not worthy in our opinion.

The limitation of this study is that it was a single-center study with a small sample size, too imprecise to draw a definite conclusion. Hence, a multi-center investigation with a larger sample size and longer follow-up duration is indispensable to find an accurate comparison. We did not evaluate the effect of External Beam Radiation Therapy (EBRT) on hematological toxicity. We only studied radiation's effect on pelvic $\mathrm{BM}$, while the rest of $\mathrm{BM}$ also contributes to the body's bone marrow reserve. Our institution is a regional cancer center catering to a high number of patients from Eastern and Southern India. Hence, we could not generate two plans SD-IMRT and BMS-RT, for each patient due to logistic reasons. However, patients with similar pathological profiles were compared. Due to the lack of FLT-PET facility in our institution, we could not include it in this investigation.

\section{Conclusion}

This study found no significant difference in DVH parameters between the conventional SD-IMRT treatment arm and the BMS-IMRT treatment arm. It is evident from this study that SD-IMRT is enough to limit the radiation dose to the bone marrow without affecting the dose to the targeted volume.

\section{Conflict of interest}

None declared.

None declared.

\section{Funding}

\section{Acknowledgment}

The authors would like to thank the team of IndQuench Life Science Innovations (OPC) Pvt.Ltd. for their constructive comments and support to develop this manuscript.

\section{References}

1. World Health Organization. Globocan 2018: Estimated cancer incidence, mortality and prevalence worldwide in 2018. http://globocan.iarc.fr/Pages/fact_sheets_cancer. aspx. (17th Sep 2020.). 
2. Arbyn $M$, Weiderpass $E$, Bruni $L$, et al. Estimates of incidence and mortality of cervical cancer in 2018: a worldwide analysis. Lancet Glob Health. 2020; 8(2): e191-e203, doi: 10.1016/s2214-109x(19)30482-6, indexed in Pubmed: 31812369.

3. Ellis RE. The distribution of active bone marrow in the adult. Phys Med Biol. 1961; 5: 255-258, doi: 10.1088/00319155/5/3/302, indexed in Pubmed: 13726497.

4. Blomlie V, Rofstad EK, Skjønsberg A, et al. Female pelvic bone marrow: serial MR imaging before, during, and after radiation therapy. Radiology. 1995; 194(2): 537-543, doi: 10.1148/radiology.194.2.7824737, indexed in Pubmed: 7824737.

5. Abu-Rustum NR, Lee S, Correa A, et al. Compliance with and acute hematologic toxic effects of chemoradiation in indigent women with cervical cancer. Gynecol Oncol. 2001;81(1): 88-91, doi: 10.1006/gyno.2000.6109, indexed in Pubmed: 11277656.

6. Sacks EL, Goris ML, Glatstein E, et al. Bone marrow regeneration following large field radiation: influence of volume, age, dose, and time. Cancer. 1978; 42(3): 10571065, doi: 10.1002/1097-0142(197809)42:3<1057::aidcncr2820420304>3.0.co;2-p, indexed in Pubmed: 100197.

7. Rubin $\mathrm{P}$, Landman S, Mayer E, et al. Bone marrow regeneration and extension after extended field irradiation in Hodgkin's disease. Cancer. 1973; 32(3): 699-711, doi: 10.1002/1097-0142(197309)32:3<699::aidcncr2820320324>3.0.co;2-v, indexed in Pubmed: 4726969.

8. Sykes MP, Chu FC, Savel H, et al. The effects of varying dosages of irradiation upon sternal-marrow regeneration. Radiology. 1964; 83: 1084-1088, doi: 10.1148/83.6.1084, indexed in Pubmed: 14226812.

9. Mauch P, Constine L, Greenberger J, et al. Hematopoietic stem cell compartment: acute and late effects of radiation therapy and chemotherapy. Int J Radiat Oncol Biol Phys. 1995; 31(5): 1319-1339, doi: 10.1016/03603016(94)00430-S, indexed in Pubmed: 7713791.

10. World Health Organization, Worldwide Prevalence of Anaemia. https://apps.who.int/iris/bitstream/handle/10665/43894/9789241596657_eng.pdf;jsessionid=72 54FDDA31F0C871E95F26EEAFE5E6CC? sequence $=1 ; 1993$ to 2005 (18th Sep 2020.).

11. Brixey CJ, Roeske JC, Lujan AE, et al. Impact of intensitymodulated radiotherapy on acute hematologic toxicity in women with gynecologic malignancies. Int J Radiat Oncol Biol Phys. 2002; 54(5): 1388-1396, doi: 10.1016/ s0360-3016(02)03801-4, indexed in Pubmed: 12459361.

12. Lujan AE, Mundt AJ, Yamada SD, et al. Intensity-modulated radiotherapy as a means of reducing dose to bone marrow in gynecologic patients receiving whole pelvic radiotherapy. Int J Radiat Oncol Biol Phys. 2003; 57(2): 516-521, doi: 10.1016/s0360-3016(03)00521-2, indexed in Pubmed: 12957265.

13. Mahantshetty U, Krishnatry R, Chaudhari S, et al. Comparison of 2 contouring methods of bone marrow on $\mathrm{CT}$ and correlation with hematological toxicities in nonbone marrow-sparing pelvic intensity-modulated radiotherapy with concurrent cisplatin for cervical cancer. Int
J Gynecol Cancer. 2012; 22(8): 1427-1434, doi: 10.1097/ IGC.0b013e3182664b46, indexed in Pubmed: 22932264.

14. Bansal $A$, Patel FD, Rai $B$, et al. Literature review with PGI guidelines for delineation of clinical target volume for intact carcinoma cervix. J Cancer Res Ther. 2013; 9(4): 574-582, doi: 10.4103/0973-1482.126450, indexed in Pubmed: 24518699.

15. Gay HA, Barthold HJ, O'Meara E, et al. Pelvic normal tissue contouring guidelines for radiation therapy: a Radiation Therapy Oncology Group consensus panel atlas. Int J Radiat Oncol Biol Phys. 2012; 83(3): e353-e362, doi: 10.1016/j. ijrobp.2012.01.023, indexed in Pubmed: 22483697.

16. Mell LK, Schomas DA, Salama JK, et al. Association between bone marrow dosimetric parameters and acute hematologic toxicity in anal cancer patients treated with concurrent chemotherapy and intensity-modulated radiotherapy. Int J Radiat Oncol Biol Phys. 2008; 70(5): 1431-1437, doi: 10.1016/j.ijrobp.2007.08.074, indexed in Pubmed: 17996390.

17. Mell LK, Kochanski JD, Roeske JC, et al. Dosimetric predictors of acute hematologic toxicity in cervical cancer patients treated with concurrent cisplatin and intensity-modulated pelvic radiotherapy. Int J Radiat Oncol Biol Phys. 2006; 66(5): 1356-1365, doi: 10.1016/j. ijrobp.2006.03.018, indexed in Pubmed: 16757127.

18. Hong L, Alektiar K, Chui C, et al. IMRT of large fields: wholeabdomen irradiation. Int J Radiat Oncol Biol Phys. 2002; 54(1): 278-289, doi: 10.1016/s0360-3016(02)02921-8, indexed in Pubmed: 12183002.

19. Rose BS, Aydogan B, Liang Y, et al. Normal tissue complication probability modeling of acute hematologic toxicity in cervical cancer patients treated with chemoradiotherapy. Int J Radiat Oncol Biol Phys. 2011; 79(3): 800-807, doi: 10.1016/j.jijrobp.2009.11.010, indexed in Pubmed: 20400238.

20. Mell LK, Schomas DA, Salama JK, et al. Association between bone marrow dosimetric parameters and acute hematologic toxicity in anal cancer patients treated with concurrent chemotherapy and intensity-modulated radiotherapy. Int J Radiat Oncol Biol Phys. 2008; 70(5): 1431-1437, doi: 10.1016/j.ijrobp.2007.08.074, indexed in Pubmed: 17996390.

21. Mell LK, Kochanski JD, Roeske JC, et al. Dosimetric predictors of acute hematologic toxicity in cervical cancer patients treated with concurrent cisplatin and intensity-modulated pelvic radiotherapy. Int J Radiat Oncol Biol Phys. 2006; 66(5): 1356-1365, doi: 10.1016/j. ijrobp.2006.03.018, indexed in Pubmed: 16757127.

22. McGuire SM, Bhatia SK, Sun W, et al. Using [(18)F] Fluorothymidine Imaged With Positron Emission Tomography to Quantify and Reduce Hematologic Toxicity Due to Chemoradiation Therapy for Pelvic Cancer Patients. Int J Radiat Oncol Biol Phys. 2016; 96(1): 228-239, doi: 10.1016/j.ijrobp.2016.04.009, indexed in Pubmed: 27319286.

23. Munshi A, Ganesh T, Mohanti BK. Radiotherapy in India: History, current scenario and proposed solutions. Indian J Cancer. 2019; 56(4):359-363, doi: 10.4103/ijc.IJC_82_19, indexed in Pubmed: 31607709. 